\title{
Maximizing Precision Over Extended Unambiguous Range for TOF Range Imaging Systems
}

\author{
Adrian P. P. Jongenelen, Dale A. Carnegie \\ School of Engineering and Computer Science \\ Victoria University of Wellington \\ Wellington, New Zealand \\ adrian.jongenelen@vuw.ac.nz
}

\author{
Andrew D. Payne, Adrian A. Dorrington \\ Department of Engineering \\ University of Waikato \\ Hamilton, New Zealand
}

\begin{abstract}
The maximum unambiguous range for time-of-flight range imaging systems is inversely proportional to the chosen modulation frequency. However, increasing the unambiguous range by decreasing the modulation frequency will generally also degrade the range measurement precision. We describe a technique that significantly extends the range of a time-of-flight imaging system without compromising range precision. This is achieved by employing two modulation frequencies simultaneously. The chosen frequencies can be a combination of high and low frequency, or two similarly high frequencies.

In this paper we present experimental results comparing single frequency; dual high and low frequency; and dual high frequency operation and demonstrate that range precision need not be appreciably compromised to achieve an extended unambiguous range.
\end{abstract}

Keywords- range imaging, ambiguity, image sensor, time-offlight, 3D camera.

\section{INTRODUCTION}

Many existing time-of-flight (TOF) range imaging systems $[1,2,3]$ employ an amplitude modulated continuous wave (AMCW) light source and image sensor mechanism. The image sensor measures the phase delay of the modulation envelope of light that is reflected from objects in the scene, allowing the time-of-flight and consequently object distance to be calculated for each pixel using

$$
d=\frac{c}{2 f_{M}}\left(\frac{\varphi}{2 \pi}+k\right)
$$

where $f_{M}$ is the modulation frequency, $\varphi$ is the phase delay, $c$ is the speed of light and $k$ is an integer. Measurement of $\varphi$ is restricted to the range $[0,2 \pi)$; however the phase delay of the returned light may exceed $2 \pi$ radians at large object distances. In this instance, the integer $k$ has a non-zero value. The variable $k$ can not be determined from a single phase measurement, and therefore is typically assumed to be zero, restricting the maximum unambiguous object range to

$$
d_{u}=\frac{c}{2 f_{M}} .
$$

Objects measured beyond this distance will be wrapped around to fall in the range $\left[0, d_{u}\right)$, appearing much closer than they actually are. The most common and simple method to extend the unambiguous range, $d_{u}$, is to reduce the modulation frequency, $f_{M}$, however this results in a proportional loss of range measurement precision. With ongoing advancement in electronic manufacturing, range imaging systems are expected to employ higher modulation frequencies to improve measurement precision; however this approach further complicates the trade off between optimizing precision and the maximum unambiguous measurement range. An alternative method involves taking two separate captures of the scene at different modulation frequencies [3]. Each measurement gives an independent set of possible positions of the target object, and these are combined by selecting which results are most in agreement.

In Section II an overview of TOF range imaging using a single modulation frequency is presented, with a discussion on the parameters which affect precision. Section III describes the potential improvements in precision to be gained through using dual frequency modulation. The concepts have been implemented and tested on an imaging system constructed by the authors, and this experimental setup is described in Section IV. Comparisons have been made between single frequency modulation and dual frequency modulation methods in terms of distance measurement precision. Particular emphasis has been placed on comparing schemes using one low and one relatively high modulation frequency versus two high modulation frequencies, and an investigation into how the ratio between time spent at the two frequencies affects precision. Results of these experiments are presented in Section IV, with a summary in Section V.

\section{Single Modulation FREQUENCY RANGING}

Indirect TOF range imaging systems utilize an AMCW light source to illuminate the scene. The light is reflected back from objects in the scene and integrated over a time period, $T_{I}$, by an image sensor gain modulated at the same frequency. Due to the time taken for the light to travel to and from the scene, the modulation phase envelope of the returned light is shifted relative to the image sensor modulation. When the two signals are in phase, the pixels within the sensor accept more photons producing a high intensity value, and the inverse is

A. P. P. J acknowledges the New Zealand Tertiary Education Commission for funding through a Top Achievers Doctoral Scholarship. 
true when the returned light and sensor modulation are out of phase.

Between captured image samples the phase of the image sensor modulation is stepped by $2 \pi / N$ radians, where $N$ is the number of image samples per range measurement. After $N$ samples have been captured, the phase, $\varphi$, magnitude, $A$ and DC offset, $B$, (partially due to ambient light) of the light returned to each pixel can be calculated using

$$
\begin{gathered}
\varphi=\tan ^{-1}\left(\frac{\sum_{i=0}^{N-1} I_{i} \sin \theta_{i}}{\sum_{i=0}^{N-1} I_{i} \cos \theta_{i}},\right. \\
A=\frac{1}{2} \sqrt{\left[\sum_{i=0}^{N-1}\left(I_{i} \cos \theta_{i}\right)\right]^{2}+\left[\sum_{i=0}^{N-1}\left(I_{i} \sin \theta_{i}\right)\right]^{2}}, \\
B=\frac{1}{N} \sum_{i=0}^{N-1} I_{i},
\end{gathered}
$$

where $I_{i}$ are the pixel intensity values read from the image sensor for frame number $i$, and $\theta_{i}$ are the phase steps between consecutive samples. Distance is then computed from the phase using (1), with a maximum unambiguous range given by (2). The standard deviation of this measurement can be modeled by [1]

$$
\sigma_{d}=\frac{c}{4 \sqrt{2} \pi f_{M}} \cdot \frac{\sqrt{B+A}}{c_{d} A}
$$

where $c_{d}$ is the modulation contrast describing the demodulation performance of the system. To improve precision the following methods can be used:

- Increase the modulation frequency, $f_{M}$;

- Increase the ratio of $\frac{A}{\sqrt{B+A}}$ either by

○ increasing the integration time;

○ increasing the power of the illumination source;

○ reducing ambient light levels;

○ or increasing the reflectivity of the objects in the scene;

- Increase the modulation contrast, $c_{d}$.

Reducing ambient light levels or increasing the reflectivity of objects in the scene is undesirable, as it restricts the operating conditions of the system. Similarly, increasing the power output of the illumination source is not always feasible in environments where eye safety is a factor. The modulation contrast is largely dependent on the image sensor itself and is difficult to improve without redesigning the sensor. This leaves modulation frequency and integration time as the two primary tools for improving system precision. It should be noted that the modulation contrast is somewhat dependent on the modulation frequency, therefore increasing the modulation frequency does not always yield improved precision. However, it may be expected that future systems benefiting from improved electronics manufacturing techniques and more advanced sensor design will see an improvement in this respect.

The resultant measurement frame rate of the system, $f_{O}$ accounts for the time taken to acquire $N$ raw image samples at a sampling rate of $f_{S}$. Acquiring each raw image frame consists of a user configurable integration period, $T_{I}$, plus a typically fixed readout period, $T_{R}$, where the pixel values are transferred out of the sensor. These are related by

$$
f_{O}=\frac{f_{S}}{N}=\frac{1}{N\left(T_{I}+T_{R}\right)} .
$$

It follows that in a situation where the measurement frame rate is to be held constant, the integration period, and hence precision can be increased by reducing $N$ where possible to minimize the sensor readout time. The standard value for $N$ is four for all current range imaging systems in use due to the relative simplicity of generating the four phase offsets using digital switching circuits and the simplification of the sine and cosine values to $\{-1,0,1\}$ in equations (3) and (4). At the cost of slightly more complicated processing, the phase, magnitude and offset values can be computed with $N=3$, allowing the integration time to be increased due to the removal of one readout period, potentially improving the range measurement precision.

\section{DUAL MODULATION FREQUENCY RANGING}

By Equation (6) measurement precision can be improved by increasing the modulation frequency, $f_{M}$. However, by Equation (2) this also decreases the maximum unambiguous range of the system. By utilizing two range results acquired with different modulation frequencies it is possible to extend the maximum unambiguous range without significantly impacting range precision $[3,4]$.

\section{A. Dual High and Low Frequency Modulation}

Fig. 1 shows how high precision can be maintained within the unambiguous range of a low modulation frequency measurement. The result $d_{l}$ is produced using a low modulation frequency with poor precision but a large unambiguous range, $d_{u l}$, where $k_{l}$ is assumed to be zero. The second phase result, $\varphi_{2}$ is produced using a modulation frequency several times higher than that of the first measurement and gives several possible object distances of $\varphi_{2} d_{u 2} / 2 \pi+k_{2} d_{u 2}$ with higher precision. Comparing these possible distances to $d_{l}$ found with the low frequency measurement, $k_{2}$ can be established as three in this example. While both results are in agreement, an unambiguous result 
with improved precision is obtained compared to a single capture at the low modulation frequency.

The major cost of this method is that twice the number of samples must be captured to obtain the unambiguous range measurement. Therefore in order to maintain the same measurement frame rate the integration period is reduced, possibly negating the precision improvement obtained with the higher frequency measurement.

In this case the low frequency measurement only contributes an estimation of the result, and therefore the precision requirement for this measurement can be relaxed. An upper limit on the precision of the low frequency measurement can be obtained by realizing that it need only be precise enough to position the object at intervals of $d_{u 2}$. Hence, it may prove beneficial to reduce the integration time of the low frequency measurement and add to the integration time of the high frequency measurement, improving the precision of the high frequency measurement while maintaining the total integration time.

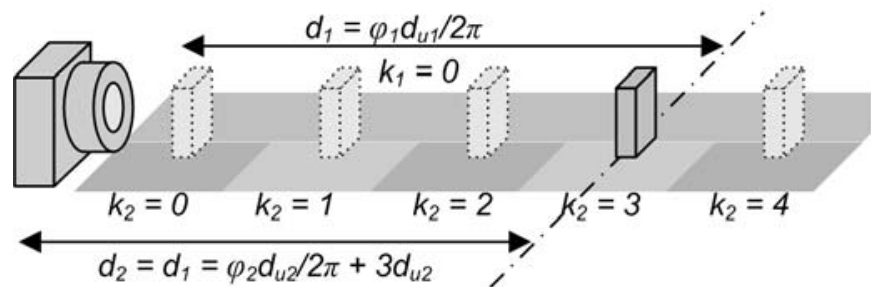

Figure 1. Measurement combining high and low frequency modulation. The low frequency measurement, $\varphi_{1}$ gives a coarse measure out to unambiguous distance $d_{u l}$, and establishes the integer $k_{2}$. The high frequency measurement, $\varphi_{2}$ is used with $k_{2}$ to find the true range with higher precision.

\section{B. Dual High Frequency Modulation}

An alternative to using low frequency operation to produce a coarse measure is to perform the second measurement also at a high, but slightly different, frequency to that of the first measurement [4]. Fig. 2 shows how this is possible.

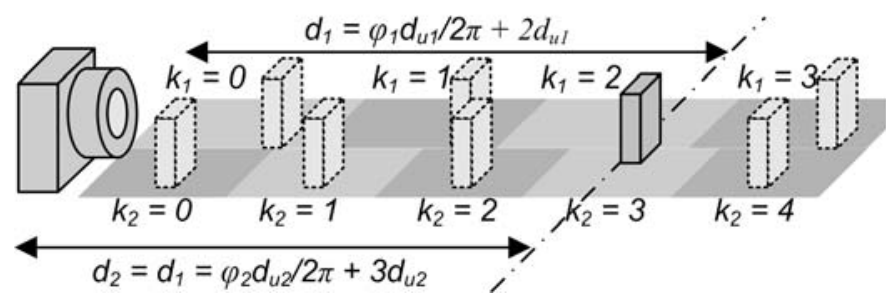

Figure 2. Measurement combining two high modulation frequencies. The true range of the object is established as the location where the two measurements are in agreement. Both measurements have high precision and can thus both contribute to the final result.

Each measurement gives a set of possible object locations, but only at one location are the two in agreement. In this case both individual results have relatively high precision and can be averaged so they both contribute to the final range measurement. The maximum unambiguous range using this method is extended to

$$
d_{u}=\frac{c}{2\left|f_{M 1}-f_{M 2}\right|} \text {. }
$$

The relative contribution from each result could be assigned as a fixed weighting of $50 \%$ or as a variable weighting based on the relative modulation frequencies and the relative signal magnitudes by the formula

$$
d_{\text {final }}=d_{1} \frac{A_{1} f_{M 1}}{A_{1} f_{M 1}+A_{2} f_{M 2}}+d_{2} \frac{A_{2} f_{M 2}}{A_{1} f_{M 1}+A_{2} f_{M 2}}
$$

where $A_{i}, f_{M i}$ and $d_{i}$ are the amplitude, modulation frequency, and calculated distance from each of the two captures.

\section{Simultaneous Dual Frequency Modulation}

The previous subsections have described methods using multiple independent captures at different modulation frequencies. We have previously demonstrated that it is also possible to encode both of these measurements simultaneously within a single capture, whereby the modulation frequency is alternated within the integration period [5]. This method utilized the dual high and low modulation frequency approach, where only the high frequency component contributed toward the final range measurement precision. The use of two independent captures at two high modulation frequencies have also been demonstrated [4,6], although the use of simultaneous modulation using multiple high frequencies appears to have not previously been reported.

In order to distinguish between the two measurements, the phase step of the second modulation frequency is doubled such that during acquisition of $N$ samples, the phase steps from 0 to $4 \pi$ radians. In effect, the first modulation frequency is sampled over one cycle (from 0 to $2 \pi$ ), while the second modulation frequency is sampled over two cycles (from 0 to $4 \pi$ ), allowing each phase and magnitude value to be resolved from the combined measurement. As there are five unknowns, the DC offset and the phase and magnitude of each modulation frequency component, a minimum of five samples are required to uniquely determine these variables; one less than the case where the two captures are performed independently. The removal of one frame readout period, $T_{R}$, increases the total integration time available for a given measurement rate, potentially improving the range measurement precision.

\section{EXPERIMENTAL SETUP}

The authors have constructed a range imaging system based on the PMD Technologies PMD19-k image sensor. Illumination is provided by a bank of eight laser diodes driven in a controlled current configuration mounted in a circular arrangement around a $16 \mathrm{~mm}$ focal length lens. The modulation signals are generated using an Altera Stratix III FPGA. Simultaneous dual frequency modulation has been implemented using phase-locked-loops within the FPGA; one for each of the two frequencies. A controller selects which modulation source reaches the output and has the ability to 
vary the ratio of time spent at each frequency during the sensor integration time.

This system exhibits an increase in precision up to a modulation frequency of $40 \mathrm{MHz}$, at which point a sharp decline in precision is noted due to bandwidth limitations of the image sensor drive electronics [7]. Additionally, the modulation contrast, $c_{d}$, is reduced as the frequency is increased, which for this system implies that distance measurement standard deviation is not strictly proportional to $1 / f_{M}$. Further details regarding this system can be found in [8].

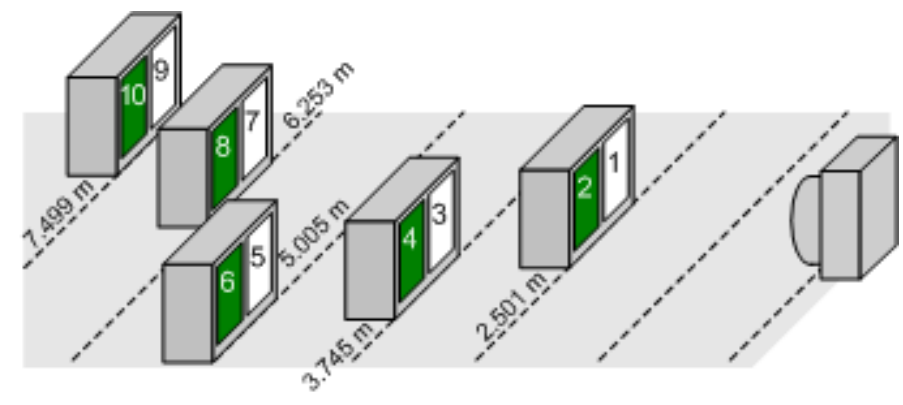

Figure 3. Diagram of the test scene. Five objects are placed at distances of approximately $1.250 \mathrm{~m}$ intervals and each object has a green and a white target.

A scene has been set up with five pairs of test targets with each pair's actual distances measured as 2.501, 3.745, 5.005, 6.253 and $7.499 \mathrm{~m}$ using a Leica DISTO distance meter with a manufacturer specified accuracy of $\pm 1 \mathrm{~mm}$. These distances have been chosen to purposely produce ambiguous distance measurements for modulation frequencies above $20 \mathrm{MHz}$. The $40 \mathrm{MHz}$ measurement is particularly interesting as ambiguity leads to some objects appearing at almost identical distances. The color of the right target in each pair is white, and the left target is green so that a comparison can be made between two objects at the same distance reflecting light of different intensities.

Initially the scene is imaged using modulation frequencies of 40 and $8 \mathrm{MHz}$ and the measurement frame rate $f_{O}$ and number of samples $N$ varied to find the effect of these parameters on the distance measurement precision.

The scene is then imaged with the system configured for simultaneous dual frequency modulation using five samples per measurement; independent dual frequency modulation using six samples per measurement (three samples for each frequency); and single frequency modulation using four samples per measurement. In this case the output measurement rate has been kept fixed at $12.5 \mathrm{~Hz}$. This rate is deemed high enough to obtain a meaningful comparison between the modulation schemes whilst still maintaining high enough accuracy to produce useful distance measurements.

To test the dual high and low frequency method the high modulation frequency, $f_{M 1}$, is selected as $40 \mathrm{MHz}$ and the low modulation frequency, $f_{M 2}$, is chosen as $8 \mathrm{MHz}$ to highlight the effect that the modulation frequency has on the measurement precision. In this case, only the $40 \mathrm{MHz}$ measurement contributes to range precision. To test the dual high frequency method, $f_{M 2}$ is selected as $32 \mathrm{MHz}$, such that when used together with the $40 \mathrm{MHz}$ measurement the maximum unambiguous range is the same as that for $8 \mathrm{MHz}$. In this case the results are combined using (9) to give the final range measurement.

For all of the sequences an $8 \times 8$ group of pixels on each target has been analyzed over 200 measurements to give an indication of the error rate. This is defined as the percentage of measurements that give an absolute range measurement error of greater than half the unambiguous range of the $40 \mathrm{MHz}$ modulation measurement. Precision is measured by calculating the 1-sigma standard deviation of all measurements that are not identified as an error above.

\section{RESULTS}

Fig. 4 shows the 1-sigma standard deviation of the measured distance for test target number 5 versus measurement period for $N$ of 3, 4 and 5 and modulation frequencies of 40 and $8 \mathrm{MHz}$. As anticipated from (6), for all measurement periods the higher $40 \mathrm{MHz}$ modulation frequency exhibits better precision. Additionally, it can be seen that better precision can be achieved by reducing the value of $N$, and that this effect is more pronounced for shorter measurement periods due to a proportionally larger pixel integration period $T_{I}$ available due to the reduction of the number of frame readout periods $T_{R}$ required within the acquisition time.

Interestingly, for this target at measurement periods longer than $100 \mathrm{~ms}$, the $8 \mathrm{MHz}$ measurement for $N=4$ outperforms $N=3$. We suspect this is due to aliased harmonics erroneously increasing the amplitude of the returned signal, thereby improving precision. It should be noted that although aliased harmonics can increase signal amplitude, they can also have a detrimental impact on measurement accuracy [7]. This effect varies with target distance, and could warrant further investigation.

Fig. 5 shows the standard deviation of the measured distance for the ten test targets using a selection of modulation schemes. For the dual frequency modulation schemes, the ratio between time spent at the two frequencies has been fixed at $50 \%$. The odd numbered targets are the white colored target of the pair, which generally exhibit improved precision compared to the green colored target at the same distance, due to the increased amplitude of the returned light signal. Targets 9 and 10 are the most distant, and exhibit the lowest precision.

In almost all cases the $8 \mathrm{MHz}$ only measurement is the least precise, despite the relatively large integration time devoted to it. The simultaneous measurements give very similar precision compared to the equivalent result if the captures are taken independently as illustrated in Fig. 5. On this particular system, the readout time is fixed at $3.2 \mathrm{~ms}$ which is relatively small compared to the total $80 \mathrm{~ms}$ measurement period used for this test. The minimal improvement achieved by increasing the integration time as described in Section III-C appears to be offset by other factors which are system specific. These factors might include extra noise within the digital electronics generating the two modulation frequencies, or the influence of harmonics from one frequency source interfering with the other. 


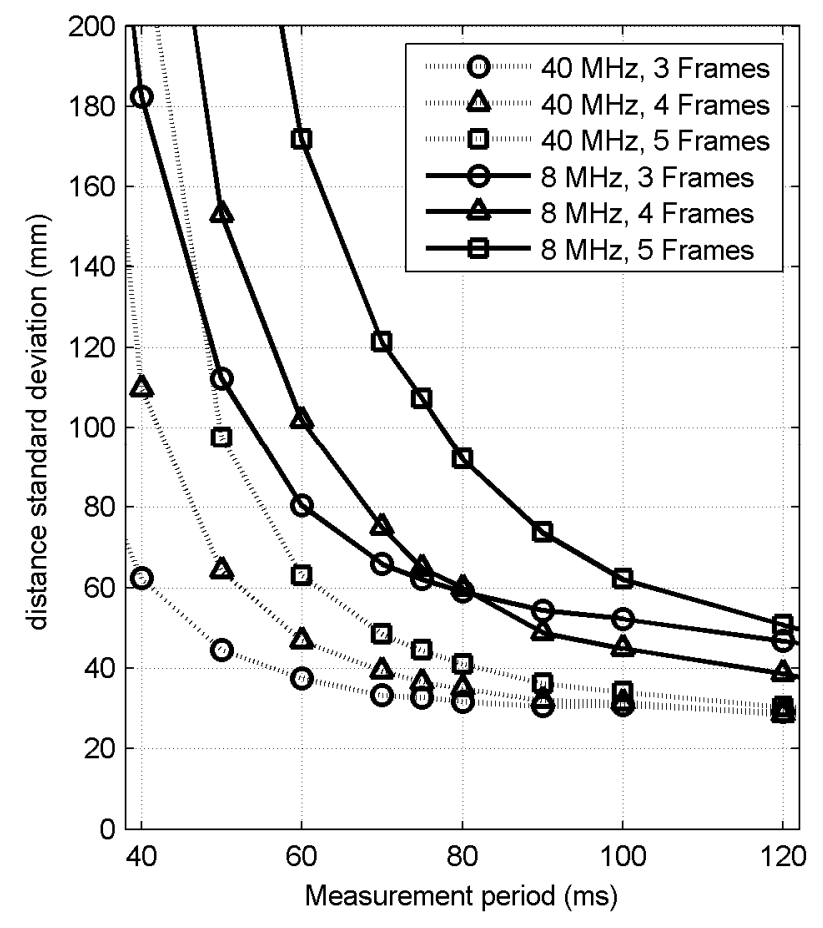

Figure 4. Distance standard deviation for test target number 5 versus integration time with $N$ of 3,4 and 5 and $f_{M}$ of 40 and $8 \mathrm{MHz}$.

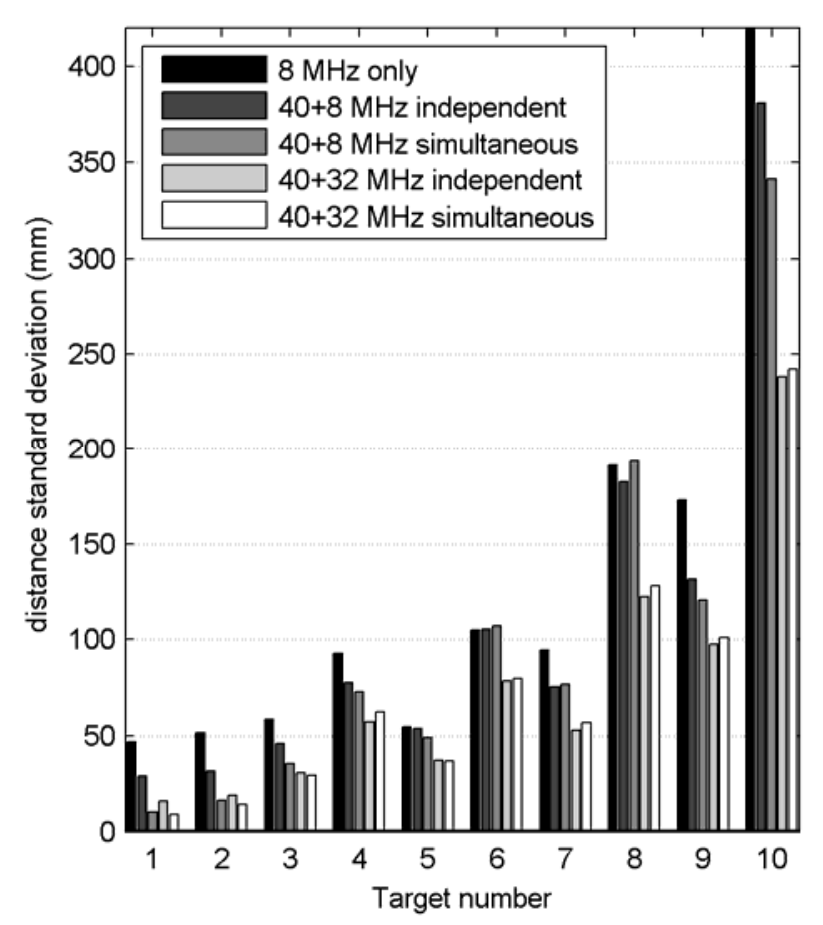

Figure 5. Standard deviation for the test target measurements using different modulation schemes.

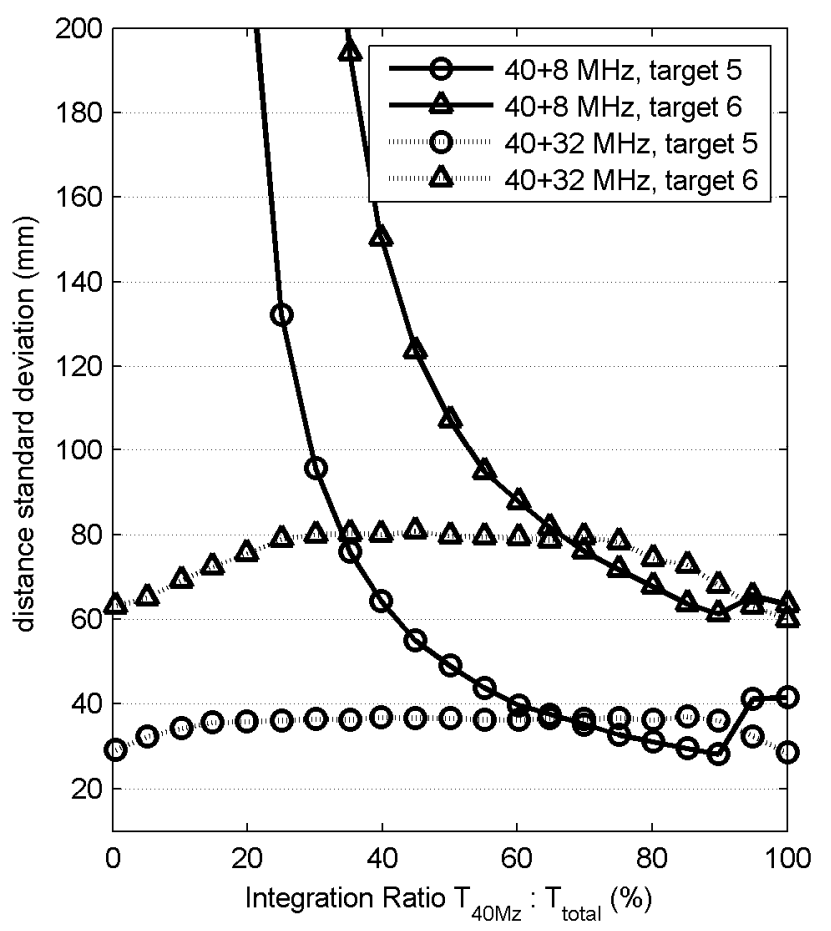

Figure 6. Distance standard deviation versus modulation ratio for two targets using high/low and high/high modulation.

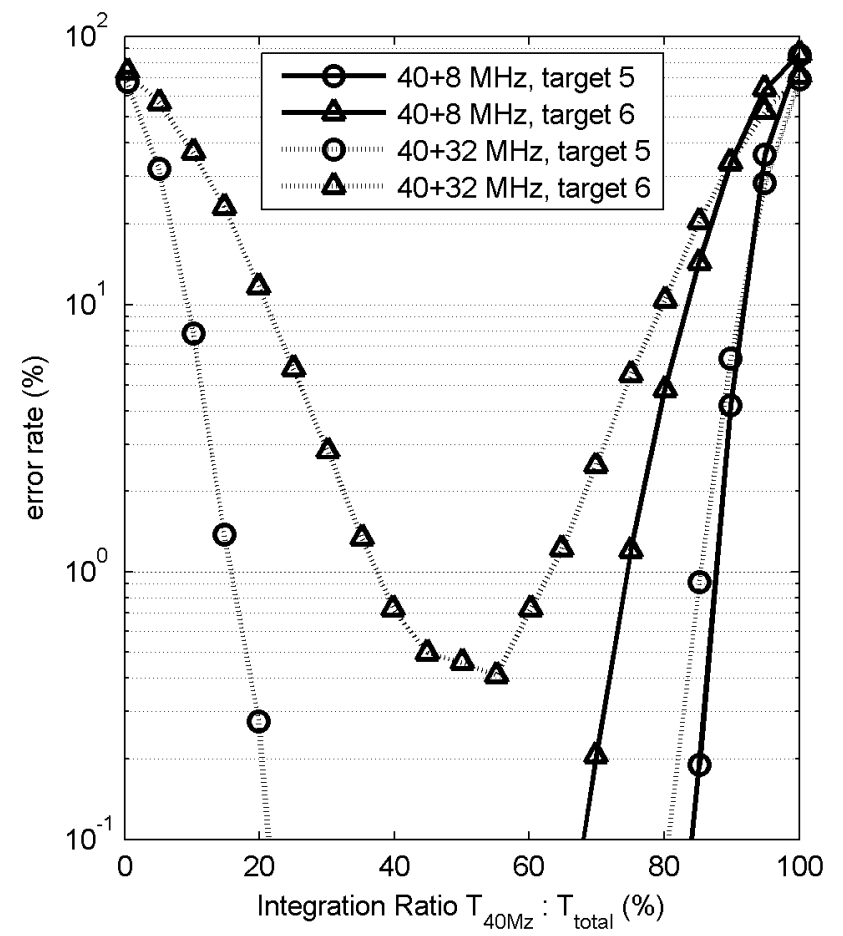

Figure 7. Error rate versus modulation ratio for two targets using high/low and high/high modulation. 
The simultaneous modulation captures were repeated, this time varying the ratio between the integration times of the two frequencies. Fig. 6 shows a plot of the measured distance standard deviation versus the integration ratio between the $40 \mathrm{MHz}$ modulation and the total integration time for the two targets $5.005 \mathrm{~m}$ distant. Fig. 7 shows the error rate versus the integration ratio for the same.

The high and low frequency measurements show the highest precision for both targets as the percentage integration time of the high frequency measurement is increased, illustrated in Fig. 6. An upper limit is reached where the low frequency result is not accurate enough to correctly determine the integer $k_{l}$, causing large errors in the range measurement as illustrated in Fig 7. With the dual high frequency measurements the precision is approximately constant, as both measurements contribute to the overall result based on a weighting determined by the magnitude and modulation frequency. However, the error rate is higher, particularly at either end of the ratio scale, since both measurements must be precise enough to correctly determine the integers $k_{l}$ and $k_{2}$.

\section{SUMMARY}

This paper has presented experimental results utilizing two measurements with different modulation frequency to measure range beyond the normal ambiguity distance if only one modulation frequency were used.

The results show that the best ratio for dual high frequency modulation is approximately $50 \%$, giving the fewest errors and highest precision independent of the reflective properties of objects in the scene. For the high and low frequency method selecting an optimum ratio between them is more complicated. While a larger ratio of the high frequency is desirable to give improved precision, without prior knowledge of the brightness of the objects in the scene it cannot be known what the minimum ratio of the low frequency measurement is required to correctly establish $k_{l}$. However, if some errors are acceptable, the high and low frequency method can provide better precision for higher ratios.

This paper has focused largely on the precision of distance measurements utilizing multiple modulation frequencies. Future work would involve determining how distance accuracy is affected by these methods. In particular, even for a single modulation frequency, the influence of harmonics introduces a systematic error which is dependant on object distance, which becomes much more complicated when using multiple frequency measurements.

\section{REFERENCES}

[1] B. Büttgen and P. Seitz, "Robust optical time-of-flight range imaging based on smart pixel structures," IEEE Trans. on Circuits and Systems I: Regular Papers, vol. 55, pp. 1512-1525, 2008.

[2] G. Gulden, D. Becker, and M. Vossiek, "Novel optical distance sensor based on MSM technology," IEEE Sensors Journal, vol. 4, no. 5, pp. 612-618, 2004.

[3] S.B. Gokturk, H. Yalcin, C. Bamji, "A Time-of-Flight Depth SensorSystem Description, Issues, and Solutions," in Proc. Computer Vision and Pattern Recognition Workshop, 2004, http://canesta.com/productsand-technology/products/technical-papers.

[4] A. A. Dorrington, M. J. Cree, A. D. Payne, R. M. Conroy and D. A. Carnegie, "Achieving sub-millimetre precision with a solid-state fullfield heterodyning range imaging camera", Measurement Science and Technology, vol. 18, pp 2809-2816, 2007.

[5] A. D. Payne, A. P. P. Jongenelen, A. A. Dorrington, M. J. Cree, and D. A. Carnegie, "Multiple Frequency Range Imaging to Remove Measurement Ambiguity," Optical 3-D Measurement Techniques IX, vol. 2, pp. 139-148, 2009.

[6] Canesta Inc., "Canesta 101 - Introduction to 3D Vision in CMOS", 2008, http://canesta.com/products-and-technology/products/technicalpapers.

[7] A. D. Payne, A. A. Dorrington, M. J. Cree, D. A. Carnegie, "Characterization of modulated time-of-flight range image sensors," Proc. SPIE-IS\&T Electronic Imaging, vol. 7239, 2008.

[8] A. P. P. Jongenelen, D. A. Carnegie, A. P. Payne, A. A. Dorrington, "Development and Characterisation of an Easily Configurable Range Imaging System", Proc. Image and Vision Computing New Zealand, 2009. 